\title{
Therapeutic efficacy of Sulfadoxine/ Pyrimethamine in the treatment of uncomplicated malaria in children
}

\author{
Solomon Worku, Tsinuel Girma, Yonas Shiferaw
}

\begin{abstract}
Background: In Ethiopia, like in most other sub Saharan Africa countries, malaria remains a major public health problem with P.falciparum accounting for the highest number of deaths. Owing to its effectiveness and low cost, treatment with Sulfadoxine/Pyrimethamine (SP) was and is still the recommended first line treatment for uncomplicated P. falciparum malaria. However, the rapid emergence of resistance is considered to be the main drawback for the use of this drug in malaria endemic areas.

Objectives: This study is aimed at assessing the extent of resistance to SP by P. falciparum malaria.

Methods: A cross sectional study, was conducted in Oct. 2003 in Jimma town. The study included children under 15 years of age who were infected with P.falciparum malaria and who have fulfilled the inclusion criteria. The patients were treated with the standard regimen of Sulfadoxine/Pyrimethamine. Clinical and parasitological responses to the drug sample were monitored for 14 days.

Results: A total of 95 children below 15 years of age were considered for the study, out of whom 86 have completed the 14-day follow up with a 9.5\% loss to follow up. The age of the children ranged from one to 14 years 60 (63.2\%) of them were males and 35 (36.8\%) were females with a male to female ratio of 1.71 . With regard to age distribution, 24 (25.3\%)were in the under-five-age category, 41(43.2\%) in the age group 5-9, and 30 (31.6) were in the age group 1014. Forty seven $(54.7 \%)$ children responded successfully both clinically and parasitologically to SP treatment. Twenty-two (25.6\%) children had late parasitological failure, and 17(19.7\%) had late clinical failure. The mean parasite count at recruitment was 32,651 per $\mu \mathrm{l}$ with a range of 2,100 $-190,000$. The period time for clearance of parasitemia was 2.7 days $( \pm 2.53 \mathrm{SD})$.
\end{abstract}

Conclusion: This study has shown that the prevalence of resistance to SP is on the increase, which may indicate the need for searching for affordable and effective substitutes to SP. [Ethiop.J.Health Dev. 2005;19(1):11-15]

\section{Introduction}

Malaria remains as the major disease of public health importance in Ethiopia; and leads the list of top diseases for outpatient morbidity. It is among the leading causes of hospitalization and hospital deaths (1).

It is also a significant health problem for many countries, and its effect has even worsened with the emergence of drug-resistant strains of the parasite.

Sulphadoxine/Pyrimethamine remains to be the only drug of choice as first line treatment for uncomplicated P.falciparum malaria in most African countries (Chloroquine was replaced by Sulphadoxine/ Pyrimethamine as first line treatment for uncomplicated P.falciparum malaria in Ethiopia in 1999) (1).

However, the rapid emergence of resistance and the slower clinical response are considered to be the main drawbacks in the use of this drug product in malariaendemic areas $(2,3)$.

Inspite of the wide use of Sulphadoxine/Pyrimethamine over the last ten years in the country, an evaluation of the efficacy of these drugs from 1996 to 1998 found the drug to be highly effective in the treatment of uncomplicated P.falciparum malaria (1). However, there is evidence that the prevalence of clinical resistance to Sulphadoxine/ Pyrimethamine in East Africa is on the increase (4).

As the trend of malaria changes over time, the efficacy and effectiveness, tolerance and safety including mild side effects, as well as severe and life threatening adverse effects of the recommended treatment should be monitored. Emphasis particularly should be given to monitoring the emergence of resistance to SulphadoxinePyrimethamine (1).

Therefore, close monitoring of resistance patterns would be required to maximize the therapeutic benefits of this drug.

No study has been conducted so far though personal observation from daily practice and informal communication with health professionals has raised doubts about the effectiveness of Sulphadoxine/ Pyrimethamine on P. falciparum malaria.

Based on this notion, this study set the objective to determine the practical efficacy of single dose Sulfadoxine/Pyrimethamine intake in the routine treatment of uncomplicated P.falciparum malaria. 


\section{Methods}

The study was conducted in children who were screened at the Malaria Control Center in Jimma town, south west of Ethiopia in Oct.2003.

The Sample size was determined to be 97 , based on a $50 \%$ expected proportion of treatment failure, $95 \%$ confidence interval and a $10 \%$ precision target (5).

After informed consent was obtained from parents or guardians, children between the age of 6 months and 14 years of age were included in the study by making user that they have fulfilled the following inclusion criteria: mono infection with Plasmodium falciparum, a parasitemia level of 2,000- 200,000/ $\mathrm{ll}$, absence of danger signs or signs of severe and complicated malaria according to the definition given by WHO (6), presence of auxillary temperature $\left(\geq 37.5^{\circ} \mathrm{C}\right)$, absence of other conditions like pneumonia which can cause fever, no use of SP two weeks prior to the study and if following visits were possible.

Blood films were taken five times for each patient during the study (day 0, 2, 3, 7, and 14). Thick blood films were dried and stained with Giemsa stain at of a PH of 7.4 and were examined to determine parasite density (100 high power field (HPF) were used and the number of asexual parasites per HPF was recorded), according to the method described in the WHO Protocol (5). Blood slide is considered negative if 100 fields on a thick film are examined and no parasites are seen. Parasite count was based on the number of asexual parasites observed against 200 leukocytes. This number was then multiplied by 40 to gain an approximate count per microlitre (parasitemia per $\mu \mathrm{l}=$ number of parasite $\mathrm{x} 8000 /$ number of leukocytes).

A case record form was completed and patients were given a single dose of Sulfadoxine/Pyrimethamine (Fansidar, Hoffman la Roche) based on body weight at a dose of $1.25 \mathrm{mg}$ per $\mathrm{Kg}$ Pyrimethamine or $25 \mathrm{mg}$ Sulfadoxine per $\mathrm{Kg}$ according to the WHO guideline (6). The dose was given under supervision and was repeated if vomiting occurred within one hour. Children with an auxillary temperature of $38.5^{\circ} \mathrm{C}$ or higher were treated with Paracetamol (15mg per kg per dose taken orally every $6 \mathrm{hr}$ for $24 \mathrm{hrs}$ ). Parents or guardians were asked to bring their children on days 1,2,3,7 and 14 for followup and or any other day if the illness worsened so that the child could be examined, temperature recorded and blood films are taken.

Oral quinine was given to children with treatment failures while those with danger signs were referred to hospitals.

The response to treatment was classified based on the WHO Protocol (6). Early treatment failure (ETF) is defined as parasitemia higher than day 0 count on day 2 irrespective of auxillary temperature $\geq 37.5^{\circ} \mathrm{C}$ or parasitemia on day $3 \geq 25 \%$ of count on Day 0 . The definition of late clinical failure (LCF) is presence of parasitemia on any day from day 4 to 14 at a measured auxillary temperature of $\geq 37.5^{\circ} \mathrm{C}$, without previously meeting any of the criteria of ETF while late parasitological failure (LPF) was defined as the presence of parasitemia on day 14 and a measured auxillary temperature of less than $37.5^{\circ} \mathrm{C}$, without previously meeting any of the criteria of ETF and LCF. Adequate clinical and parasitological response (ACPR) is defined as the absence of parasitemia on day 14 irrespective of auxillary temperature without previously meeting the criteria for early treatment or late clinical failure or late parasitological failure.

The data were checked for completeness and the double entry system was employed while entering the data. Chi- Square and lifetime table analysis methods were used. SPSS 11.0 for windows was applied for the analysis.

Twenty percent of the blood slide samples were counterchecked for validity of parasitic count by a senior laboratory technologist.

\section{Result}

A total of 95 children below 15 years of age were considered for the study, out of whom 86 have completed the 14-day follow up with a $9.5 \%$ loss to follow up but their data was used during analysis using the life table method. The entry characteristics are shown in Table 1.

The age of the children ranged from 1 to 14 years. There were 60 (63.2\%) males and 35 (36.8\%) females with a male to female ratio of 1.71 . With regard to age distribution, 24 (25.3\%) were in the age group of less than five years, $41(43.2 \%)$ were in the age group of 5-9, and $30(31.6 \%)$ were in the age group of 10-14. All patients come from Jimma Zone, 56.8\% lived in Jimma town while the remaining resided in surrounding rural kebeles (Table 1).

Table 1: Baseline characteristics of children with uncomplicated $P$. falciparum malaria, Jimma town, South west Ethiopia, Oct. 2003

\begin{tabular}{ll}
\hline Characteristics & \\
\hline Number of patients & 95 \\
Age (in years) & $7.3(1.14)$ \\
Male (\%) & 63 \\
Parasitmia (per $\mu \mathrm{l})$ & $32651(2100-190000)$ \\
\hline
\end{tabular}

Using the WHO clinical treatment response criteria, 47 (54.7\%) children were found to have a successful response both clinically and parasitologically to SP treatment during the 14 day follow up period. Twentytwo (25.6\%) children had late parasitological failure, 
17(19.7\%) had late clinical failure and none of the children had early treatment failure. (Table 2)

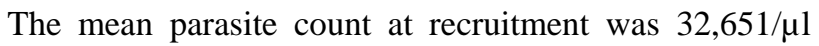
with a range of 2100 to 190,000 and the mean time for clearance of parasitemia was 2.7 days $( \pm 2.53 \mathrm{SD})$. (Fig.1)

The rate of clearance was the same in all the age groups and followed the same pattern of decrease in mean parasitic count in the first few days after treatment and an increase in the $7^{\text {th }}$ and $14^{\text {th }}$ days in those who failed to respond (Fig. 1).
Table 2: Patterns of $P$. falciparum resistance to SP, Jimma town, South west Ethiopia, Oct. 2003

\begin{tabular}{cll}
\hline & $\mathrm{N}=86$ & $\%$ \\
\hline Resistance & 39 & 45.3 \\
ETF & 0 & 0.0 \\
LCF & 17 & 19.7 \\
LPF & 22 & 25.6 \\
ACPR & 47 & 54.7 \\
\hline ETF $=$ Early Treatment Failure \\
LCF = Late Clinical Failure \\
LPF = Late Parasitological Failure \\
ACPR = Adequate Clinical and Parasitological Response
\end{tabular}

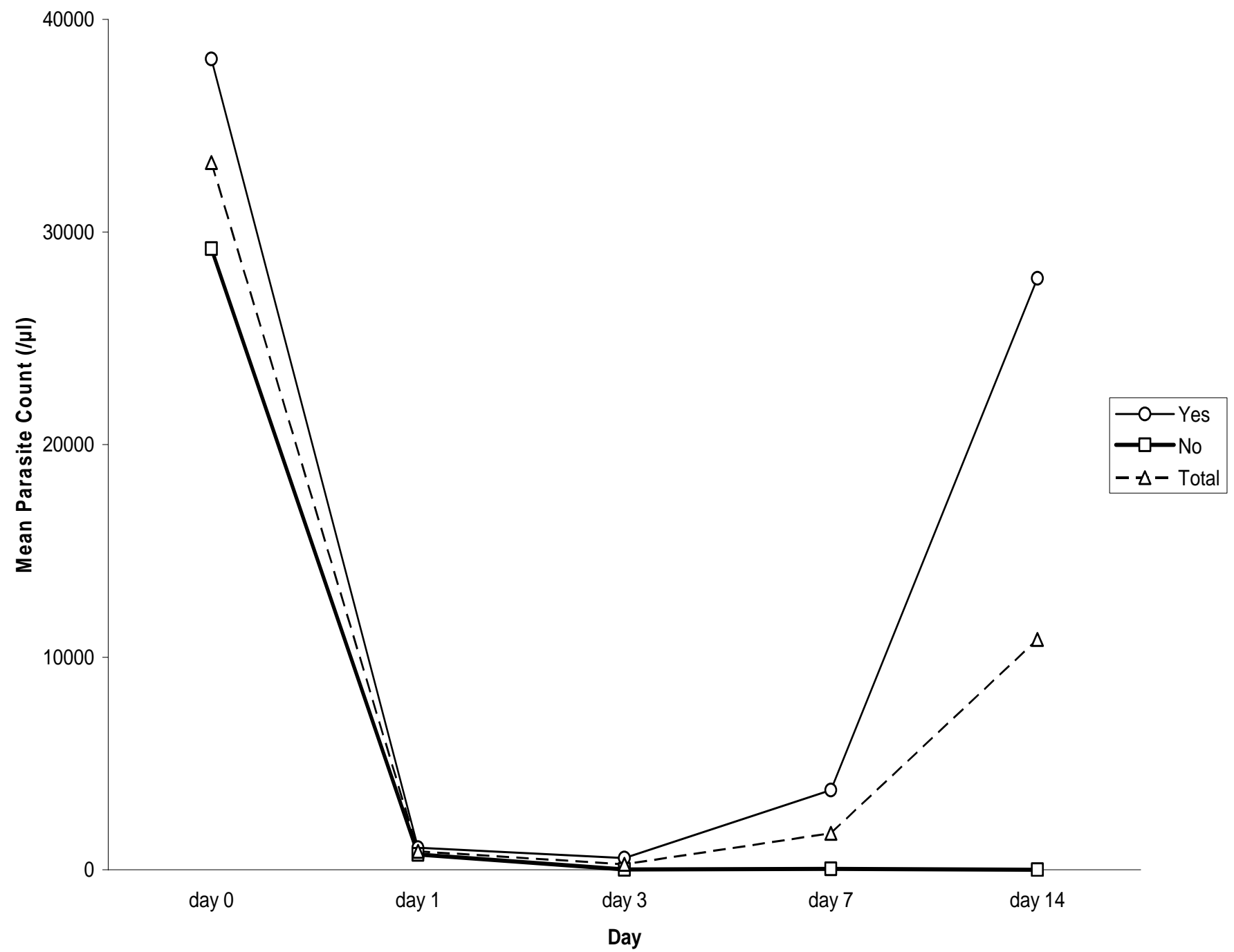

Figure 1: Mean parasite count in relation to pattern of resistance, Jimma town, South west Ethiopia, Oct. 2003

\section{Discussion}

The mean parasite count at recruitment was 32,651 per $\mu \mathrm{l}$ with a range of 2,100 $-190,000$, which is lower than a finding reported in a study in the Kenya, which showed the mean parasite count to be 44,741 and with the range 720 to 240,000 per $\mu \mathrm{l}$. But, the mean time required for the clearance of parasitemia is somehow close to the Kenyan study, which had reported 2.5 days.(4)

Sulfadoxine and Pyrimethamine are long but slow acting blood schizonticides with the estimated half lives of 148- 
216 hrs and 80-95 hrs respectively which explains the long time required for the clearance of parasitemia (7).

The rate of parasitic clearance was the same in all the age groups and followed the same pattern of increase in parasitimia in the $7^{\text {th }}$ and $14^{\text {th }}$ days in those who failed to respond. Age, sex and parasitic load didn't show statistically significant association with the response to treatment ( $p>0.05)$ unlike the case of a study in Somalia that reported that the degree of initial parasitimia seemed to influence the clinical outcome and degree of parasitimia at day $0(8)$.

This study documents that a good number of parasitological failures, (about $20 \%$ and $40 \%$ of patients) had demonstrable parasite levels in their peripheral blood at $7^{\text {th }}$ and $14^{\text {th }}$ day respectively, showing that clinical criteria alone may underestimate the rate of treatment failure.

Thirty-nine (45.3\%) children had late treatment failure in our study unlike a similar prospective observational study conducted in Kenya in 1999 that found out a lower treatment failure (6.7\% early treatment failure (ETF) and only $1.3 \%$ late treatment failure (LTF) (4).

This finding may prove the assertion that the prevalence of clinical resistance to sulfadoxine /pyrimethamine in East Africa is on the increase (4).

According to a study in Uganda, treatment failure based on clinical criteria occurred in 13 of $131(10 \%)$ patients on Sulfadoxine /Pyrimethamine treatment, nine of 131 (7\%) on amodiaquine, and four of the 138 (3\%) on the amodiaquine /sulfadoxine/ pyrimethamine. Based on parasitological criteria, treatment failed in $26 \%, 16 \%$ and $10 \%$ of these patients respectively (9).

On the contrary, based on the WHO modified 14-day in vivo drug efficacy test for uncomplicated P. falciparum malaria, 4 (6\%) of 67 Sulfadoxine /Pyrimethamine treated patients had therapeutic failures (2 ETFs and 2 LTFs) in areas of the Pacific Coast region of Colombia. The relatively low level of therapeutic failures to Sulfadoxine /Pyrimethamine compared with other South American countries raised further questions regarding factors that might have prevented the rapid development of in vivo resistance to this drug combination (10).

According to a study done in Somalia on the treatment of uncomplicated P. falciparum infection with Sulfadoxine Pyrimethamine, $98 \%(n=50)$ of the patients were reported to have achieved an adequate clinical response despite a parasitological failure rate of $76 \%$ RII/ RIII. They concluded that doubts about the therapeutic life of Sulfadoxine /Pyrimethamine in relation to malaria are raised by high levels of resistance in the study area and underline the need to identify suitable alternatives (8).
A study done on 405 Gambian children with uncomplicated P.falciparum found out a combination of Fansidar (Sulfadoxine /Pyrimethamine) plus Chloroquine was a more effective symptomatic treatment than Fansidar given alone, but neither the parasite cure rate nor the mean packed cell volume (PCV) was enhanced by use of the combination (11).

A similar study done in central Ethiopia has suggested that the combination of Sulfadoxine/Pyrimethamine and Chloroquine is safer, effective and superior in fever clearance rate and useful to delay parasite resistance to SP (12).

Another study stated the higher prevalence of SP treatment failure associated gametocytemia may contribute to increased transmission of antifolate resistant strains and further cautions against the use of Sulfadoxine/Pyrimethamine as monotherapy (13).

As to the Somali study, the high degree of parasite resistance (76\% RII/RIII) was explained by the absence of a functioning treatment policy and regulatory systems; Sulfadoxine/Pyrimethamine was made easily accessible in Somalia even for rural communities. Drug pressure associated with the wide spread use of sulfa- based antibiotics, e.g. Cotrimoxazole for the treatment of bacterial infections; might have added to the problem. The study concluded that clinical failure following the use of Sulfadoxine/Pyrimethamine can be expected sooner or later and that there is a clear need for looking beyond SP and identifying other suitable alternatives (8).

Finally, Sulfadoxine/Pyrimethamine may no longer be considered adequate for treating P.falciparum in the study area as high level of resistance is documented which underlines the need to identify suitable alternatives.

Therefore, alternative drugs like Dihydroartemisinine, Halofantrine, Mefloquine and combination therapies such as chlorproguanil-dapsone, atovaquone-proguanil and Artemisia annua herbal tea may have to be tested to avert the looming crisis.

\section{Acknowledgements}

We would like to thank Jimma University for providing the found for the research the study children and their parents, all malaria control staff, especially Wzo. Almaz Demissie and Ato Mergia, for their help with the microscopy, Ato Gebru Mulugeta for counter checking the blood slides, and Ato Tibebu Kassahun for editing the manuscript.

\section{References}

1. MOH. Guideline for malaria diagnosis and treatment for frontline health workers in Ethiopia, Epidemiology and AIDS Control Department,

Ethiop.J.Health Dev. 2005;19(1) 
Malaria and other Vectorborn Disease Control Unit. July 1999.

2. Oniyorah E, Boele van Hensbroek M, Jah MS and Greenwood B. Early clinical failure after pyrimethamin/sulphadoxine treatment of uncomplicated falciparum malaria. Trans. Roy. Soc. Trop. Med. Hyg. 1996; 90: 307 -308

3. Teklehaimanot A., Chloroquine resistant Plasmodium falciparum malaria in Ethiopia. The Lancet. 1986; 2: 127-129

4. Ogutu BR, Smoak BL, Nduati RW, Mbori-Ngacha DA, Mwatlhe F and Shanks GD. The efficacy of Fansidar in the treatment of uncomplicated malaria in Kenyan children. Trans. Roy. Soc. Trop. Med. Hyg. 2000; 4: 83-84

5. WHO. Assessment of Therapeutic efficacy of antimalarial drugs for uncomplicated P.falciparum malaria in areas with intense transmission. Geneva, Switzerland. 1996. WHO/MAL/96.1077.

6. WHO. Monitoring antimalarial resistance: Report of WHO consultation. Geneva, Switzerland. Dec 2001. WHO/CDS/CSR/EPH/2002.17.

7. Goodman and Gilman, The Pharmacological basis of Therapeutics $9^{\text {th }}$ ed., McGraw-Hill Companies, USA, 1995 ; 974, 1061

8. Warsame M, Abdillahi A, Duale ON et al. Therapeutic efficacy of Chloroquine and SP against P. falciparum infection in Somalia. Bull World Health Org. 2002; 80 (9): 704-708.
9. Staedke SG, Kamaya MR, Dorsey G et al. Amodiaquine, sulfadoxine /pyrimethamine and combination therapy for treatment of uncomplicated falciparum malaria in Kampala, Uganda. The Lancet. 2001; 358: 368-374.

10. Osorio LE, Giraldo LE, Grajales LF, et al Assessment of therapeutic response of P.falciparum to Chloroquine and Sulfadoxine /Pyrimethamine in an area of low malaria transmission in Colombia, Am J Trop Med Hyg. 1999;61(6):968-72.

11. Bojang KA et al. A trial of Fansidar plus chloroquine or fansidar alone for the treatment of uncomplicated malaria in Gambian children. Trans Roy Soc Med Trop Hyg. 1998;92:72-76.

12. Moges K, Mengesha T, Mekonnen Y, Mohammed $\mathrm{H}$, Balcha F, Bulcha S. A randomized trial of Sulfadoxine/Pyrimethamine plus Chloroquine versus SP alone for the treatment of uncomplicated P. falciparum malaria in children, central Ethiopia, Ethiop. Pharm J. 2001; 19: 9-19.

13. Tjitra E, Suprianto S, Anstey NM. Higher gametocyte prevalence following failure of treatment of Plasmodium falciparum malaria with SP and the combination of CQ/SP-implication for progression of antifolate resistance. Trans Roy Soc Trop Med Hyg. 2002; 96: 434-37. 
Medical Sciences Faculty, Jimma University, P.O. box 343, Jimma, Ethiopia, Email: solomonwb@yahoo.com 